\title{
ABC Algorithm Based Interval Type-2 Fuzzy Logic Controller for an Inverted Pendulum
}

\author{
Anita Khosla \\ EEE Dept, Faculty of Engineering and Technology, Manav Rachna International University, Faridabad, India \\ E-mail: emailanitakhosla.fet@mriu.edu.in \\ Leena G. \\ EEE Dept, Faculty of Engineering and Technology, Manav Rachna International University, Faridabad, India \\ E-mail: leenag.fet@mriu.edu.in \\ M. K. Soni \\ Faculty of Engineering and Technology, Manav Rachna International University, Faridabad, India \\ E-mail: ed.fet@mriu.edu.in
}

\begin{abstract}
In this paper, a hybrid control technique is proposed for managing the variation of angle and velocity of the inverted pendulum. The proposed hybrid technique is the combination of $\mathrm{ABC}$ algorithm and interval type-2 Fuzzy Logic System (IT2FLS). The objective of the proposed hybrid control technique is to achieve the stability position of the pendulum. Here, the $\mathrm{ABC}$ algorithm is used to optimize the change of angle and change of velocity of the pendulum. With the optimized value, the optimal membership functions and the interference system are developed using IT2FLS. Using the ABC based IT2FLS, the position of the inverted pendulum is maintained towards the reference position. The proposed hybrid control technique is implemented in MATLAB/Simulink working platform and the control performances are evaluated.
\end{abstract}

Index Terms - Inverted Pendulum, Angle, Velocity, ABC Algorithm and IT2FLS

\section{INTRODUCTION}

The cart type inverted pendulum system is a mu ltivariable,nonlinear, fast reaction, unstable and high order system. It is a classical control problem that is used by many researchers to design and test control technique. $[1,2]$.

For an inverted pendulum cart, a number of research works previously exist in the literatures that make use of control techniques.

A number of types of controllers are accessible for designing, analyzing and controlling the inverted pendulum both linear and non-linear dynamic deviations. Like PI controller, fuzzy controller, optimal controller and etc. However, these kinds of controllers are incompetentas they need a large nu mber of equations and control methods. For linear control problem only the PI controller is appropriate. The PI increase tuning is one of the complex problems in nonlinear control problem. For controlling the nonlinear dynamic difference of the system, the fuzzy logic controller is a rule base control method that is used. Although, in rule base controller, the rules obstruction problem has to be happened that affected the control presentation of the system. Moreover, these kinds of controller are controlled by only one control parameters (arm angle, pendulum angle, arm velocity and pendulum velocity) of the system. In order to control the angle and velocity variation of the inverted pendulum, a hybrid control technique is proposed in this paper. The proposed technique is detailed in following section.

The rest of the paper is organized as follows. In section II modeling of inverted pendulum is shown. Proposed hybrid controller for improving velocity and angle of inverted pendulum is discussed in section III. It also includes detailed discussion of $\mathrm{ABC}$ algorithm to optimize the pendulum angle and velocity and how to controlthe pendulum angle and velocity using IT2FLC with the complete procedure. In section IV results and discussion are given with performance analysis of change in angle and velocity of Inverted Pendulum. Finally conclusion is presented in section VI.

\section{MODELING OF INVERTED PENDULUM}

The inverted pendulum system is one of the examples which commonly used in control system application. The reputation of the drives in part from the fact that it is unstable without control. Because, the pendulum will simply fall over if the cart isn't moved to balance it. In addition, the dynamic states of the system are nonlinear. The intention of the control system based controller is to balance the inverted pendulum by applying a force to the cart that the pendulum is attached. [3]. It consists of an inverted beam (pole) on a moving cart as shown in Figure1. The task of the controller is to stabilize the pole angle $(\theta)$ and the cart position (x) by applying the force 
(F). The configuration and the free body diagram are illustrated in Figure1(a) and Figure1(b) respectively.

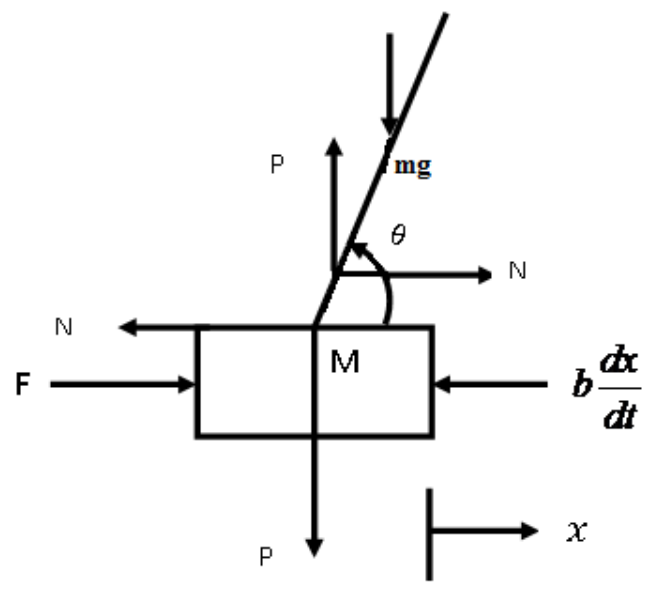

Fig. 1(a). Configuration of Inverted pendulum

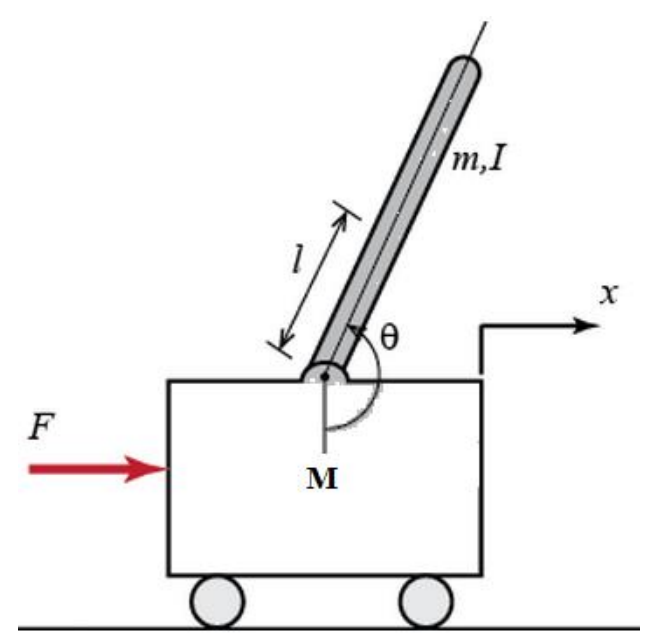

Fig. 1(b). Free body diagram

where $\mathrm{F}$ is the control (applied force), $\mathrm{x}$ is the displacement of the cart, theta is the angle of the with respect to the vertical axis, $(\Delta \theta)$ indicates the pendulum deviation position from the equilibrium position i.e. ; $\mathrm{g}=9.8 \mathrm{~m} / \mathrm{s} 2$ is gravity acceleration. Other parameters are the mass of the cart $\mathrm{M}=0.455 \mathrm{Kg}$, mass of the pole $\mathrm{m}=0.21 \mathrm{Kg}$; the friction coefficient $\mathrm{b}=$ $0.1 \mathrm{~N} / \mathrm{m} / \mathrm{sec}$; distance to pole centre of mass $1=0.3 \mathrm{~m}$; the inertia of the pole $\mathrm{I}=.0035 \mathrm{Kg} . \mathrm{m} 2$ The governing equation of the system is described as follows,

$$
\begin{aligned}
& m l \frac{d^{2} x}{d t^{2}}=\left(I+m l^{2}\right) \frac{d^{2} \phi}{d t^{2}}-m g l \phi \\
& (M+m) \frac{d^{2} x}{d t^{2}}+b \frac{d x}{d t}-m l \frac{d^{2} \phi}{d t^{2}}=0
\end{aligned}
$$

The above mathematical derivations are used to model inverted pendulum. For controlling the angle and the velocity of the pendulum, the IT2FLS is used.

The proposed hybrid controller structure shown in Figure 2 is used for controlling the angle and the velocity of the pendulum. An artificial bee's colony (ABC) algorith $\mathrm{m}[3]$ is used for optimizing the input me mbership function of fuzzy inference system.

\section{PROPOSED HYBRID CONTROLLER FOR IMPROVING THE VELOCITY AND ANGLE OF INVERTED PENDULUM}

Initially, the angle and velocity of the inverted pendulum are evaluated. After that, the change in the velocity and the change in angles are determined by taking the difference between the present and earlier error. By applying these changes of angle and change of velocity as the input to the IT2LS, the control output is calculated. Before giving the inputs to the IT2FLS, the change of angle and the change of velocity are optimized by using $\mathrm{ABC}$ algorithm and the optimized members hip functions are generated from the obtained optimized output of ABC algorithm. [4] The objective function of $\mathrm{ABC}$ algorithm is depends on the control output of the IT2FLS. The proposed hybrid controller block diagram is illustrated in fig 2. The detailed description of the proposed hybrid technique is explained in below section.

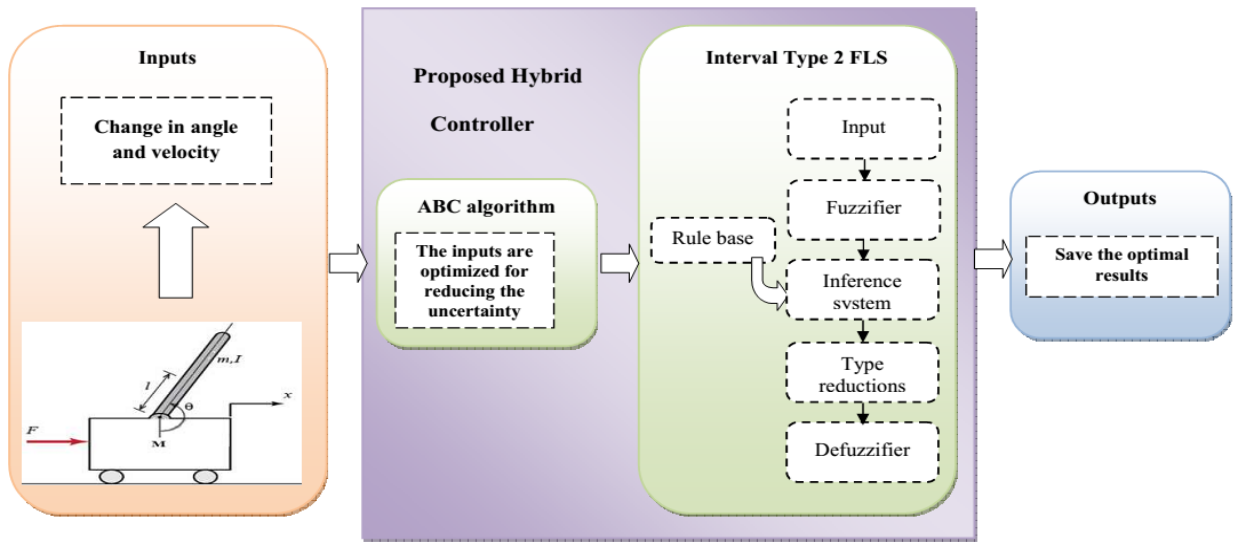

Fig. 2. Structure of proposed hybrid control model 


\subsection{Using ABC Algorithm to Optimize the Pendulum Angle and Velocity}

$\mathrm{ABC}$ algorithm is a swarm based meta-heuristic algorith $\mathrm{m}[4]$ which was stimulated by the sharp foraging behavior of the honey bees. Employed bees, onlooker bees and scout bees are the three components of $\mathrm{ABC}$ algorithm. The number of food sources indicates the position of possible solutions of optimization problem and the nectar amount of a food source indicates the excellence of the solution [5]. In this section part, the change of angle $(\Delta \theta)$ and the change of velocity $(\Delta v)$ of the pendulum are optimized by using ABC algorithm. The optimized inputs are applied to the IT2FLS and the optimized membership function is generated. The procedures of the $\mathrm{ABC}$ algorithm is specified as follows,

\section{Steps for ABC algorithm}

\section{Step 1: Initial phase}

The change of angle $\left(\Delta \theta_{i}\right)$ and velocity $\left(\Delta v_{i}\right)$ of inverted pendulum are considered as the parameters of the food sources in the population, which are generated arbitrarily. The random number of the population is $X_{i} \in\left(\Delta \theta_{i}, \Delta v_{i}\right)$, where $i=1,2, \ldots . N$ and $\mathrm{N}$ denotes the size of population. This generation process is called as initialization process.

\section{Step 2: Fitness Function}

To evaluate the optimal parameter of the inverted pendulum, the fitness value is calculated using equation (1),

$$
\text { Fitnessfunction } F_{i}=\min \left(\Delta \theta_{i}, \Delta v_{i}\right)
$$

After that, the phase of the employed bee is carried out.

\section{Step 3: Employed bee phase}

Update the velocity and angles of pendulum positions using the following equation,

$$
Y_{i, j}=X_{i, j}+\phi_{i j}\left(X_{i, j}-X_{k, j}\right)
$$

Where, $Y_{i, j}$ is the new value of the $j^{\text {th }}$ position and $k$, $j$ is a random selected index. Here, $\phi$ is randomly produced the number in the range $[-1,1]$.

Then evaluate the fitness values and apply the greedy selection between them $Y_{i, j}$ and $X_{i, j}$. Determine the probability values for the solutions $X_{i, j}$ by means of their fitness values using the following equation,

$$
p_{i}=\frac{F_{i}}{\sum_{i=1}^{d} F_{i}}
$$

Where $p_{i}$ is the probability of the $i^{\text {th }}$ parameter value.

\section{Step 4: Onlooker bee phase}

Generate the new solutions or positions $Y_{i}$ for the onlookers from the solutions $X_{i}$, selected depending upon the probability value $p_{i}$ and calculated them. Then, the fitness function is calculated for the new solutions. In order to select the best value, apply greedy selection for the onlooker bee between $X_{i}$ and $Y_{i}$.

\section{Step 5: Scout bee phase}

In this section, the abandoned solution, if exist, and replace it with a new randomly produced solution.

Memorize the best food source position achieved. The particular parameter value has been selected as the optimal value. The above process is repeated until the maximum iteration is reached otherwise the process is terminated. The flowchart for the proposed ABC algorithm is illustrated in Fig. 3.

\subsection{Controlling the Pendulum Angle and Velocity Using IT2FLS}

For stabilizing the inverted pendulum model, the fuzzy logic controller [6] is to be considered. Here, IT2FLS is used for In the IT2FLS, the rule base part enclosed with antecendents and consequents parts and each rule inference output is a type-2 fuzzy set.

The membership function limits are chosen with the help of the optimal velocity and the controlling the inverted pendulum angle and the velocity with minimum control deviation.

T2FIS is a fuzzy logic system, which contains fuzzifier, rule base, inference engine, type reductions and defuzzifier [7].optimal angle[8]. It is used for reducing footprint of uncertainties (FOU)[9]. A uniform weighting is supposed to symbolize a FOU since the mean changeable between $\mathrm{m} 1$ and $\mathrm{m} 2$, the gaussian function is measured with a standard deviation. The structure of the propsoed IT2FLS is given in figure 4. 


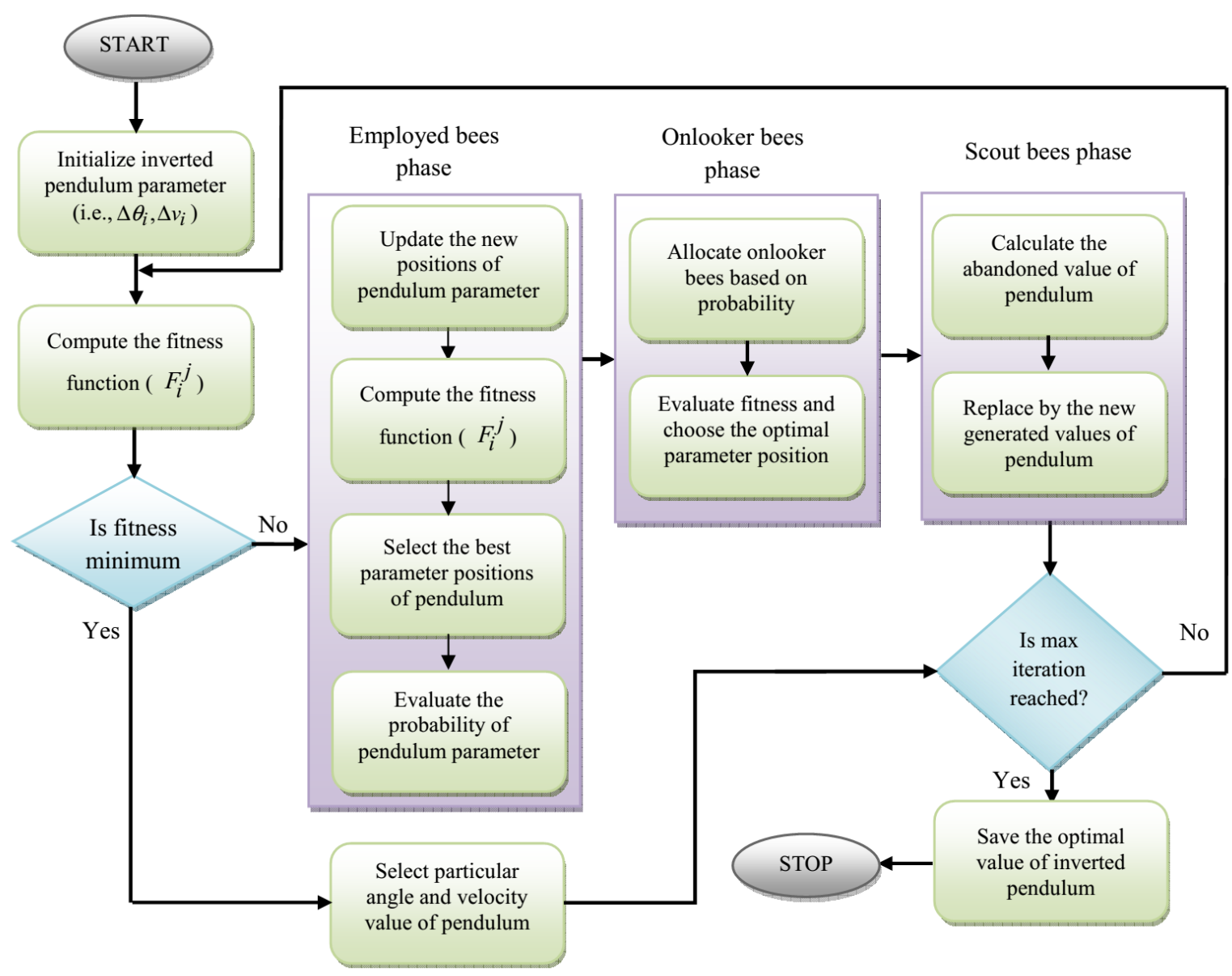

Fig. 3: Flowchart of the $\mathrm{ABC}$ algorithm for optimizing the inverted pendulum parameters deviation

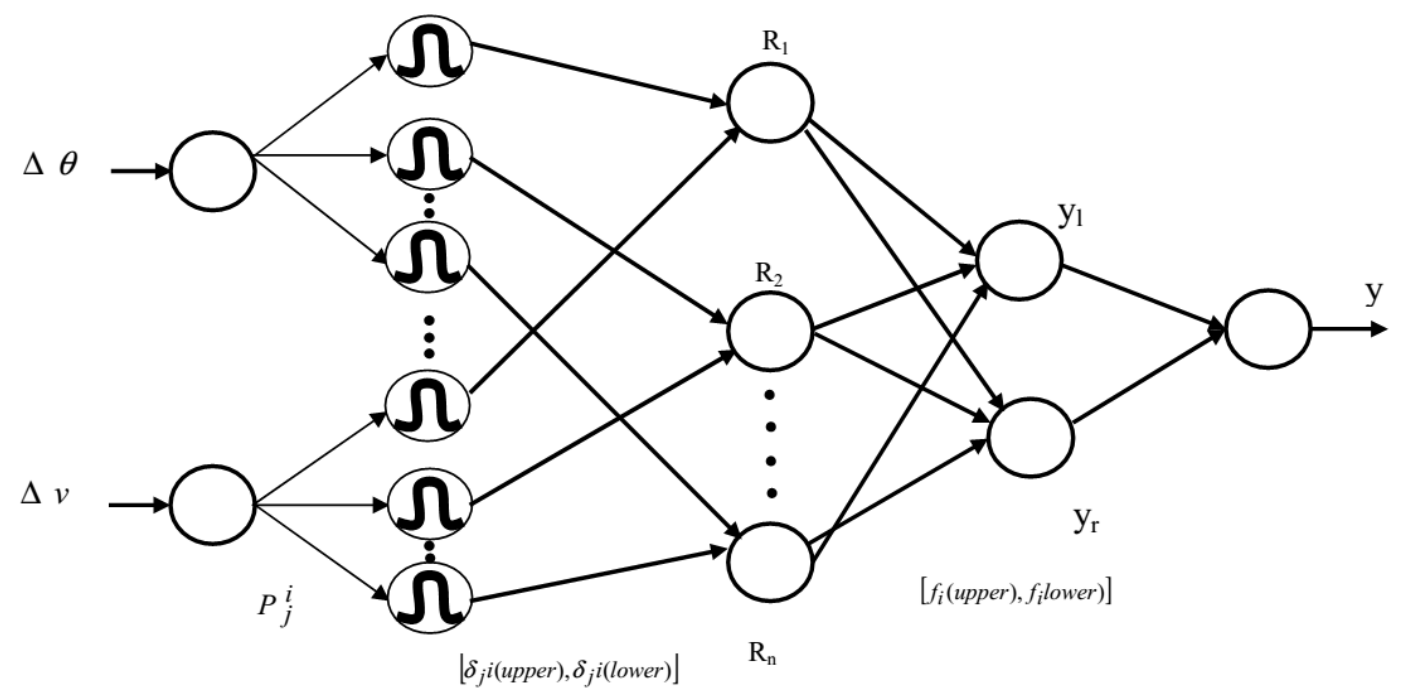

Fig. 4: Structure of proposed IT2FLS

\subsection{Procedure of IT2FLS}

\section{Step 1: Fuzzification}

\section{Fuzzification}

It is the first step of the IT2FLS, which converts the crisp parameters of input $\Delta \theta$ and $\Delta v$ are fuzzified into input interval type-2 fuzzy sets. In fuzzification, the change of angle and change of velocity are maps to the 
linguistic labels of fuzzy sets. [10] The membership functions of these fuzzy sets contain two inputs and one output. Here, the input vector is $X_{i}=\left(x_{1}, x_{2}, \ldots \ldots x_{n}\right)$ and the if-then rules are specified in the following,

$$
\begin{gathered}
R l_{i}=\text { If } x_{i} \text { is } P_{1}^{i} \text { AND } x_{2} \text { is } P_{2}^{i} \ldots \ldots \ldots . . . \\
\text { AND } x_{n} \text { is } P_{n}^{i}
\end{gathered}
$$

Then $Y_{i}$ is $a_{i}$

Where, $P_{j}^{i}$ are the antecedents $j=1,2,3 \ldots . . n$ and $Y_{i}$ is the consequent of the $i^{\text {th }}$ rule. Then the Gaussian membership function (MF) is used which has a fixed standard deviation (SD) and uncertain mean that takes in the values in $\left\lfloor m_{j 1}, m_{j 2}\right\rfloor$. The uncertainty of this membership functions can be represented in the bounded interval in terms of upper MF and lower MF. The Gaussian membership functions with uncertain mean described as,

$$
\delta_{j}^{i}\left(x_{j}\right)=\exp \left(\frac{-\left(x_{j}-m_{j}^{i}\right)^{2}}{2\left(D_{j}^{i}\right)^{2}}\right)
$$

Where, $m_{j}^{i} \in\left[m_{j 1}, m_{j 2}\right]$ and $j=1,2,3 \ldots . n, n$ is the number of antecedents. Then the $i=1,2,3 \ldots . r, r$ is the number of rules. Then the upper and lower MF's are specified as $\delta_{j} i$ (upper) and $\delta_{j} i$ (lower) respectively and described in the (8) and (9).

$$
\delta_{j} i \text { (upper) }=\left\{\begin{array}{l}
T\left(m_{j 1}^{i}, D_{j}^{i} ; x_{j}\right) \quad x_{j}<m_{j 1}^{i} \\
1 \quad m_{j 1}^{i} \leq x_{j} \leq m_{j 2}^{i} \\
T\left(m_{j 1}^{i}, D_{j}^{i} ; x_{j}\right) \quad x_{j}>m_{j 2}^{i}
\end{array}\right.
$$

and

$$
\delta_{j} i(\text { lower })=\left\{\begin{array}{cc}
T\left(m_{j 2}^{i}, D_{j}^{i} ; x_{j}\right) & x_{j} \leq \frac{m_{j 1}^{i}+m_{j 2}^{i}}{2} \\
T\left(m_{j 1}^{i}, D_{j}^{i} ; x_{j}\right) & x_{j}>\frac{m_{j 1}^{i}+m_{j 2}^{i}}{2}
\end{array}\right.
$$

Where,output of the each node is represented as in the lower and upper interval.

\section{Step 2: Rule base system}

Here, the fuzzy meet operation is done by generating the rule $[11,12]$. Two rules are generated by adding the upper membership functions seperately and lower membership function seperately for each node. The output of a rule node is a firing strength $F S^{i}$, which is an IT2 fuzzy set. The firing strength is computed as follows,

$$
F^{i}=\left(f_{i}(\text { lower }), f_{i}(\text { upper })\right)
$$

Where,

$$
\begin{aligned}
& f_{i}(\text { upper })=\prod_{j=1}^{n} \delta_{j}^{i}(\text { upper }) \\
& f_{i}(\text { lower })=\prod_{j=1}^{n} \delta_{j}^{i}(\text { lower })
\end{aligned}
$$

\section{Step 3: Inference Engine}

After obtaining the firing strength, the inference engine and the rule base are activated by the interval type- 2 fuzzy sets to produce output. Then the input interval type-2 fuzzy sets and output interval type- 2 are mapped by combining the fired rules using inference engine. Then outcome of the inference engine are processed by the type-reducer. The type-reducer combines the output sets and performs a centroid calculation which leads to type-1 fuzzy sets called the type-reduced sets.

\section{Step 4: Type Reductions}

The type reduced set of the interval type-2 FS is an interval type 1 set which contains the left limit yl and right limit $y r[13]$. The interval output of this layer is computed by each node present in this layer. The consequent $a_{i}$ is a crisp value. Here we used a simplified type-2 reduction operation instead of the center-of-sets type reduction method. It considers only two embedded type-1 fuzzy sets with membership values, that is the outputs $y^{q}$ and $y^{s}$ are computed as follows,

$$
y^{q}=\frac{\sum_{i=1}^{M} f_{i}(\text { lower }) a_{i}}{\sum_{i=1}^{M} f_{i}(\text { lower })}
$$

$$
y^{q}=\frac{\sum_{i=1}^{M} f_{i}(\text { upper }) a_{i}}{\sum_{i=1}^{M} f_{i}(\text { upper })}
$$

After the type-reduction process, the type-reduced sets are then defuzzified.

\section{Step 4: Defuzzification}

Defuzzification is the last process of the IT2FLS where each output node corresponds to one output variable. Due 
to the output layer of an interval set, each and every node performs defuzzification process[14]. The defuzzified output is denoted by $\mathrm{y}$ which is determined by taking the average of the nodes $y^{q}$ and $y^{s}$.

$$
y=\frac{y^{q}+y^{s}}{2}
$$

Subsequently the obtained defuzzified control output of fuzzy system is given to inverted pendulum to reduce the deviations of the angle and the velocity of the pendulum and also the position of the pendulum are maintained that was close to the reference position.

\section{RESULTS AND DISCUSSION}

The proposed hybrid technique was implemented in MATLAB/simulink working platform. The performance of the proposed hybrid technique was tested with inverted pendulum cart system. By using ABC algorithm, the optimal velocity and the angle parameters of the pendulum was determined. Here, the change of velocity and angles are calculated from their difference between the present state error and the previous error. This error function was set as a fitness function of the $\mathrm{ABC}$ algorith $m$. The minimum value of the fitness function is evaluated. After that, the optimized outputs are given to the IT2FLS. In the IT2FLS, the uncertainties are reduced and the optimal membership functions are determined. By using IT2FLS, the control output is generated for the inverted pendulum, which is controlling the angle and the velocity also, the stability position of the pendulum are determined. The performances of the proposed hybrid technique are evaluated.

\subsection{Performance Analysis}

Initially, the angles and velocity of the inverted pendulum performances are evaluated in normal position and illustrated in fig 5(a) and 5(b) respectively. Then applying the $\mathrm{ABC}$ algorithm, the input of IT2FLS is optimized in terms of control output 12]. The output of ABC-IT2FLS is applied to the pendulum for controlling the angle and velocity. Then, the angle and velocity of the pendulum is observed after applying the ABCIT2FLS. After that, the performances of the proposed method are evaluated and illustrated in the fig 6(a) and 6(b).

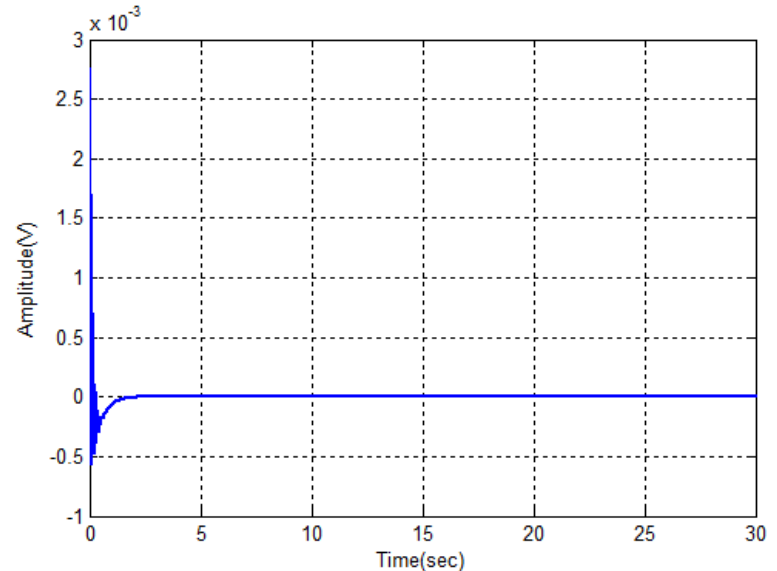

Fig. 5(a): Performance of change of angle of inverted pendulum

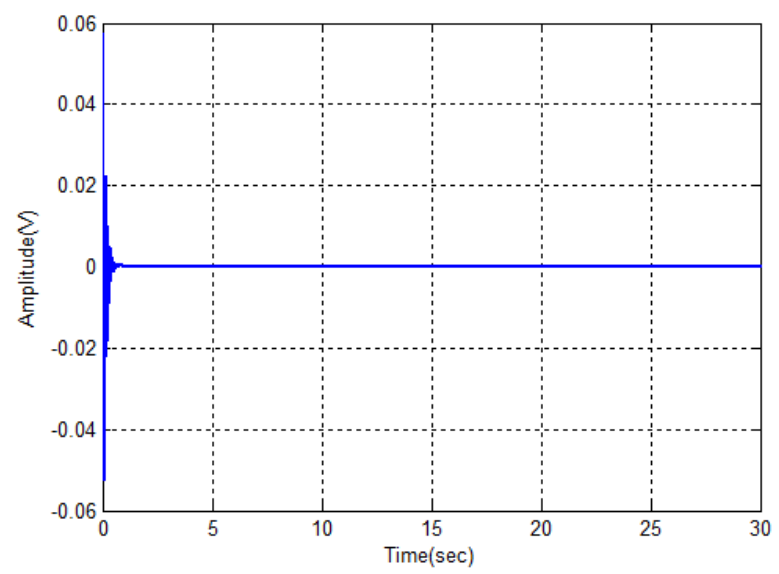

Fig. 5(b): Performance of change of velocity of inverted pendulum

These are the performances of change of angle and change of velocity with respect to time in normal position.Next performances using hybrid controller are shown.

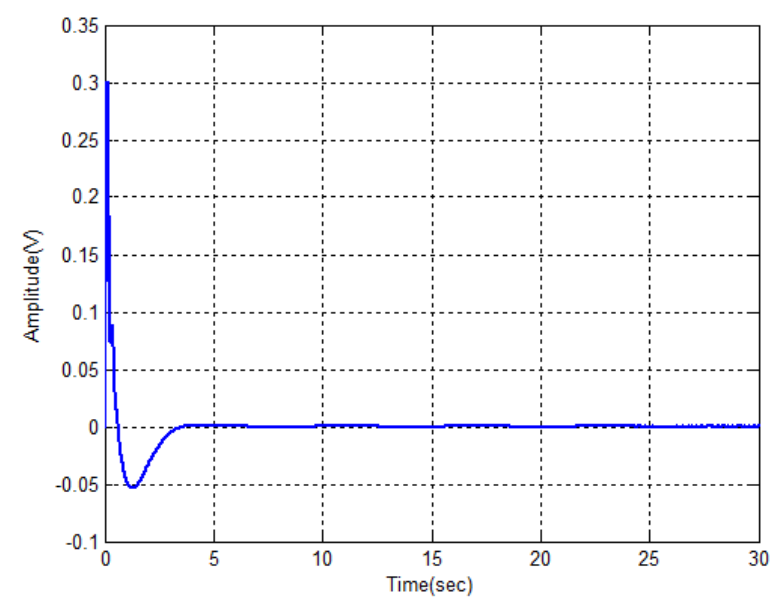

Fig. 6(a): Performance of pendulum angle using proposed hybrid controller 


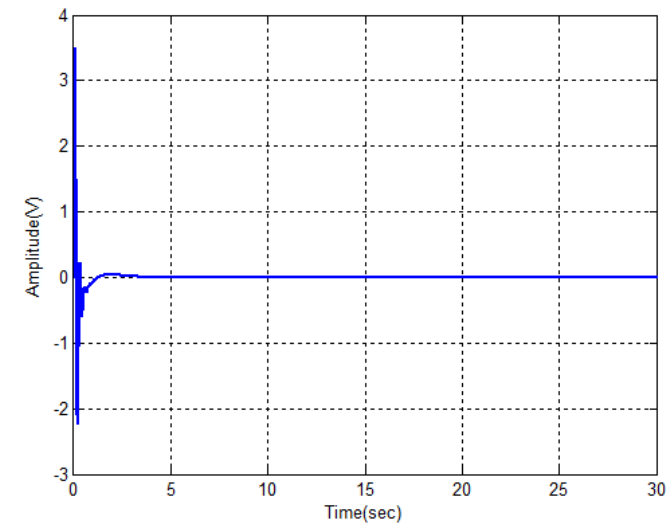

Fig. 6(b): Performance of pendulum velocity using proposed hybrid controller

From the above illustrations, the optimal value of pendulum angle and velocity are measured with the different time instants. Here, the various methods are used to calculate the optimal value of angle and velocity. The proposed hybrid controller achieves less time for optimizing the pendulum angle and velocity.

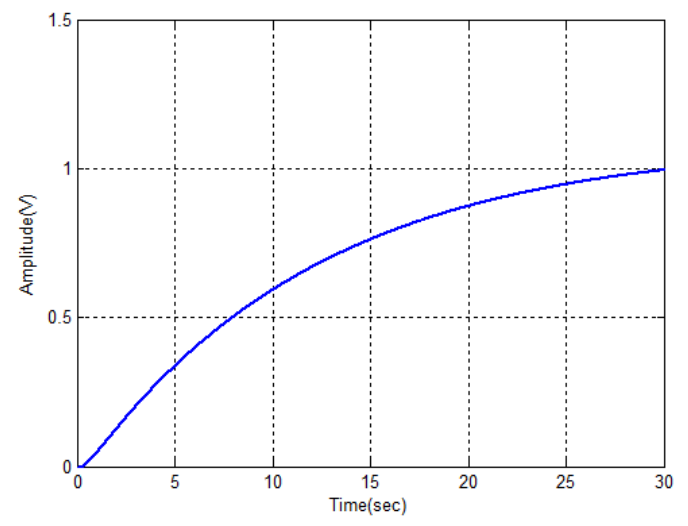

Fig. 7: Performance of normal cart position



Fig. 8: Performance of cart position after applying proposed hybrid controller

The performance of the normal cart position is illustrated in the figure7. A fter apply ing hybrid controller the cart positions is shown in fig 8 respectively.
Here, the peak value and peak time is not varied for most of these methods. The settling time of the proposed controller is $2.9800 \mathrm{sec}$. So, the proposed controller reaches the settling time to take very less time. The proposed method reaches the actual system state very quickly. Also, the proposed control technique maintains the systemstability while changing the system mass.
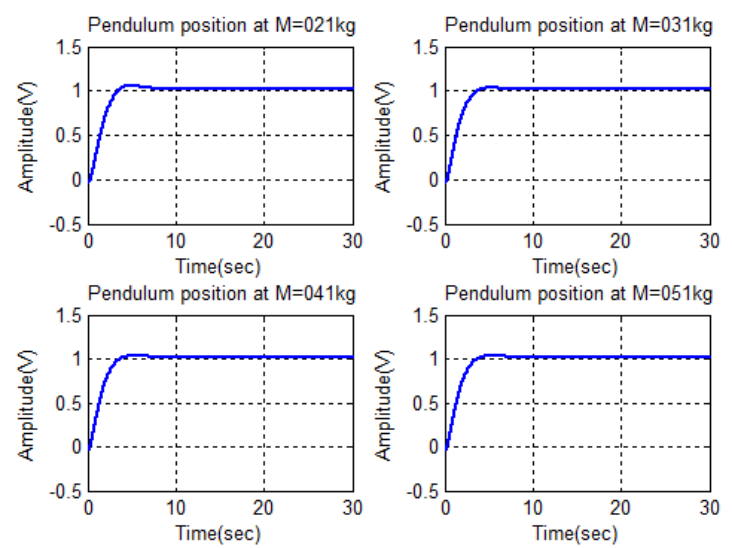

Fig. 9: Performance of cart position using proposed hybrid controller at $\mathrm{M}=0.21 \mathrm{Kg}, 0.31 \mathrm{Kg}, 0.41 \mathrm{Kg}$, and $0.51 \mathrm{Kg}$

By using the proposed hybrid method, the stability cart position of the pendulum is analyzed. The performance of cart position after applying the proposed hybrid control approach is described in Figure 8 . Then, the stability of the proposed controller is analyzed at different pendulum mass values that are $M=0.21 \mathrm{~kg}$, $0.31 \mathrm{~kg}, 0.41 \mathrm{~kg}$ and $0.51 \mathrm{~kg}$ respectively. The performance of cart position at different pendulum mass value is described in Fig 9. Therefore, the stability of the cart position is steadily preserved in the proposed hybrid method

\section{CONCLUSION}

In the paper, a hybrid control technique was proposed for controlling the angle and velocity of the inverted pendulum. The proposed hybrid technique was implemented in the MATLAB/Simulink platform. The $\mathrm{ABC}$ algorith $\mathrm{m}$ was used to optimize the change of angle and change of velocity of the inverted pendulum. By the optimized values, the range of fuzzy membership function was selected. Hence, the control performance of the IT2FLS controller is improved and reduced the uncertainties. Also, the performance of the proposed controller is evaluated at different pendulum mass values.

\section{REFERENCES}

[1] Henrik Niemann and Jesper Kildegaard Poulsen, "Analy sis and Design of Controllers for a Double Inverted Pendulum", In Proceedings of the American Control Conference Denver, Colorado, June 2003. 
[2] Shubhobrata Rudra and Ranjit Kumar Barai, "Robust Adaptive Backstepping Control of Inverted Pendulum on Cart System", International Journal of Control and Automation, Vol. 5, No. 1, March 2012.

[3] Mustafa Demirci, "Design Of Feedback Controllers For Linear System With Applications To Control Of A Double-Inverted Pendulum", International Journal of Computational Cognition, Volume 2, Number 4, Pages 65-84, December 2004.

[4] Anita Khosla, Leena G and M. K. Soni, "ABC Algorithm Based Fuzzy Controller to Control the Velocity and Angle of an Inverted Pendulum", European Journal of Scientific Research, Vol.110, No.4, pp.493-500, 2013

[5] Dervis Karaboga and Bahriye Basturk, "Artificial Bee Colony (ABC) Optimization Algorithm for Solving Constrained Optimization Problems", Foundations of Fuzzy Logic and Soft Computing, pp.789-798, 2007

[6] Xin Xin, "Analysis of the Energy Based Swing-up Control for a Double Pendulum on a Cart", In Proceedings of the 17th World Congress on the International Federation of Automatic Control Seoul, Korea, July 2008.

[7] K.J. AsstroKm and K. Furuta, "Swinging up a pendulum by energy control", International Journal of Automatica, Vol. 36, pp.287-295, 2000.

[8] Yong Xin, Bo Xu, Hui Xin, Jian Xu, and Lingyan Hu, "The Computer Simulation and Real-Time Control for the Inverted Pendulum System Based on PID", Communication Systems and Information Technology, Vol.100, pp.729-736, 2011

[9] D. Guida, F. Nilvetti and C.M. Pappalardo, "Dry friction of bearings on dynamics and control of an inverted pendulum", Journal of Achievements in Materials and Manufacturing Engineering, Vol. 38, Issue 1, January 2010.

[10] Lee, C.C., "Fuzzy logic in control systems: fuzzy logic controller. II", IEEE Transactions on Systems, Man and Cybernetics, Vol.20, No.2, pp.419-435, 1990.

[11] Ondrej Linda and Milos Manic, "On the Accuracy of Input-Output Uncertainty Modeling with Interval Type-2 Fuzzy Logic Systems", In proceedings of IEEE conference on Fuzzy systems, pp.1-7, 2012.

[12] Hung-Ching Lu, Ming-Hung Chang and Cheng-Hung Tsai, "Adaptive self-constructing fuzzy neural network controller for hardware implementation of an inverted pendulum system", Journal of Applied Soft Computing, Vol.11, pp.3962-3975, 2011

[13] Arafat Zaidan , Bashir M.Y.Nouri and Basim Alsayid, "Swing Up a Pendulum by Energy Control", International Journal of Engineering and Technology, Vol.2, No. 3, pp.528-534, March 2012.

[14] Yong Xin, Bo Xu, Hui Xin, Jian Xu, and Lingyan Hu, "The Computer Simulation and Real-Time Control for the Inverted Pendulum System Based on PID", Communication Systems and Information Technology, Vol.100, pp.729-736, 2011

\section{Authors' Profiles}

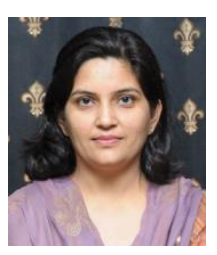

Ms. Anita Khosla has B-Tech in Electrical Engg. From NIT Kurukshetra and M-Tech in ECE in 1993and 2007 respectively. She is pursuing her $\mathrm{PhD}$ in control systems from Manav Rachna International University, India. She had over 18 years of teaching experience and presently she is working as Associate Professer in the Electrical and Electronics
Department of Manav Rachna International University, Faridabad, India.

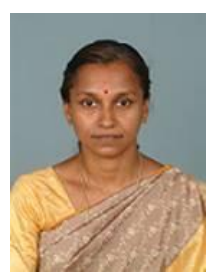

Dr. Leena G. has B-Tech in Electrical and Electronics Engg. and M-Tech in Control Systems from Kerala University in 1991and 1995 respectively. She completed her PhD in 2007 from Indian Institute of Technology, Kharagpur, India. She had over 15 years of teaching experience and presently she is heading the Electrical and Electronics Department of Manav Rachna International University, Faridabad, India. Her areas of interest are nonlinear system, decentralized control, sliding mode control etc.

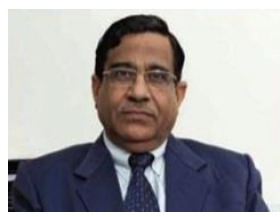

Dr. M.K.Soni has B.Sc.Engg in Electrical and MSC. Engg (Advanced Electronic \& Control System)from R.E.C. Kurukshetra in 1972 and 1975 respectively. He completed his Phd. from R.E.C Kurukshetra in collaboration with IIT Delhi in 1988.He has experience of 39 years which includes teaching, research and administration. At present he is working as Executive Director \& Dean of Manav Rachna International University, Faridabad, India. He has also worked as Director Principal of C.R.State college of Engg Murthal and as Professor \& Chairman in NIT Kurukshetra. His area of interests are Microprocessor, Microcontroller and Control Systems. Dr. Soni has guided 39 M.Tech Dissertation and and $7 \mathrm{PhDs}$. Presently $7 \mathrm{PhDs}$ are under progress under his guid ance. Dr. Soni is life time member of ISTE, Sy stem Society of India\& IASTED(Indian association of Science and Technology for Development).He is also Senior member of IEEE society.

How to cite this paper: Anita Khosla, Leena G., M. K. Soni,"ABC Algorithm Based Interval Type-2 Fuzzy Logic Controller for an Inverted Pendulum", International Journal of Intelligent Systems and Applications(IJISA), vol.6, no.6, pp.2936, 2014. DOI: $10.5815 / \mathrm{ijisa} .2014 .06 .03$ 